\title{
Two-Time Correlation Functions: Stochastic and Conventional Quantum Mechanics
}

\author{
L. Feligion:* \\ Physics Department, Boston University, Boston MA 02215 USA \\ O. Panellat \\ Istituto Nazionale di Fisica Nucleare, \\ Sezione di Perugia, Via A. Pascoli, Perugia 06123, Italy \\ Y.N. Srivastava \\ Dipartimento di Fisica dell'Università di Perugia, \\ and INFN Sezione di Perugia, Via A. Pascoli, Perugia 06123, Italy
}

\author{
A. Widom \\ Physics Department, Northeastern University, Boston MA 02115 USA
}

(Dated: October 15, 2005, - quant-ph/0202045-)

\begin{abstract}
An investigation of two-time correlation functions is reported within the framework of (i) Stochastic Quantum Mechanics and (ii) conventional Heisenberg-Schrödinger Quantum Mechanics. The spectral functions associated with the two-time electric dipole correlation functions are worked out in detail for the case of the hydrogen atom. While the single time averages are identical for stochastic and conventional quantum mechanics, differences arise in the two approaches for multiple time correlation functions.

PACS numbers: 03.65.-w, 03.65.Ta, 03.65.Ud, 01.70.+w
\end{abstract}

*Electronic address: lorenzo@bu.edu

$\dagger$ Electronic address: orlando.panella@pg.infn.it 


\section{INTRODUCTION}

While most working physicists pay homage to the Copenhagen interpretation of the Heisenberg-Schrödinger quantum mechanics (QM), many others seek a more causal reinterpretation. One ambitious effort in this direction has its origin in the works of David Bohm [1, 2]. Bohm employed a formalism for computing the paths for quantum mechanical particles closely analogous to the method of Hamilton and Jacobi. Nevertheless the Bohm approach [3, 4, 5], also known as Stochastic Quantum Mechanics (SQM), has been thought to reproduce in all instances the same probability distributions as does QM.

The research concerning SQM involves a considerable number of authors dealing with various aspects, even if not always within the terms as originally proposed by Bohm 6]. Studies of extension of the Bohm approach to the relativistic case are also available [7, 8, 9, 10, 11]. Notwithstanding the relatively difficult nature of SQM computations, it is a generally accepted belief that, where a comparison is possible, SQM and QM would give the same results. This is indeed the case for the average values of observables at a fixed time.

However, in a previous work [12], examples were reported in which SQM produces results different from QM. The examples involved the two-time correlation functions of the electric dipole moment components in the hydrogen atom. Within SQM, an explicit numerical calculation was performed 12] yielding the related spectral function (Fourier transform of the two-time correlation function) for the hydrogen atom in the excited state $|n l m\rangle=|211\rangle$. It was found to be quite different from the corresponding quantity in QM.

Our purpose is to study in more detail the comparison between the frequency spectral functions in the two theories. The general definition of two time correlation functions (for a generic system described by a time independent Hamiltonian $H$ ) is

$$
\Phi^{A B}\left(t, t^{\prime}\right)=\frac{1}{2}\left\langle A(t) B\left(t^{\prime}\right)+B\left(t^{\prime}\right) A(t)\right\rangle .
$$

The related noise spectral functions are defined as the Fourier transform

$$
S^{A B}(\omega)=\int_{-\infty}^{+\infty} e^{i \omega t} \Phi^{A B}(t) \frac{d t}{2 \pi} .
$$

Sum rules for the functions $S^{A B}(\omega)$ are investigated in terms of their $k^{\text {th }}$-order moments $\gamma^{(k)}$

$$
\gamma^{(k)}=\int_{-\infty}^{+\infty} \omega^{k} S^{A B}(\omega) d \omega
$$


Taking up (for definiteness) the case of the electric dipole moment $\boldsymbol{p}(t)=e \boldsymbol{r}(t)$ of the hydrogen atom, the following issues are addressed: (i) An explicit analytic form is derived (in terms of modified Bessel functions) within SQM for the noise spectral function of the dipole moments for the first few excited states, explicitly $|n l m\rangle=|211\rangle,|322\rangle,|321\rangle$ and $|311\rangle$. General formulas are given for special combinations of quantum numbers, e.g. $|n, l, m\rangle=$ $|n, n-1, n-1\rangle$ and $|n, n-1, n-2\rangle$. (ii) A general proof is given concerning the asymptotic behavior of the spectral functions of the dipole moment fluctuations. As $\omega \rightarrow \infty$, the spectral functions vanish with a power law for both QM and SQM, but with different exponents. (iii) The moments of the spectral functions are investigated both for QM and SQM, showing explicitly the second order moment differences.

In Sec. II a brief review of the Stochastic Quantum Mechanics is provided and in Sec. III the general notion of the two-time correlation function is defined. The related spectral function is also introduced. In Sec. IV, an explicit calculation is reported for the hydrogen atom two-time correlation function of the electric dipole moment. The spectral function asymptotic behavior for large frequency is discussed. Sec. V contains a discussion of moment sum rules, and in the concluding Sec. VI the differences between QM and SQM are further explored.

\section{THE STOCHASTIC QUANTUM MECHANICS AND PARTICLE TRAJEC- TORIES}

In SQM the wave function $\psi(\boldsymbol{r}, t)$ entering into the Schrödinger equation,

$$
i \hbar \frac{\partial \psi}{\partial t}=H \psi, \quad H=-\frac{\hbar^{2}}{2 \mu} \nabla^{2}+V
$$

is conveniently written in the form $\psi=R e^{i S / \hbar}$. $R$ and $S$ are real functions. SQM then provides a causal interpretation for the two resulting coupled differential equations thus obtained; i.e.

$$
\begin{aligned}
\frac{\partial S}{\partial t}+\frac{|\boldsymbol{\nabla} S|^{2}}{2 \mu}+V-\left(\frac{\hbar^{2}}{2 \mu}\right) \frac{\nabla^{2} R}{R} & =0, \\
\frac{\partial\left(R^{2}\right)}{\partial t}+\nabla \cdot\left(\frac{R^{2} \boldsymbol{\nabla} S}{\mu}\right) & =0 .
\end{aligned}
$$

The first of the above two equations is of the Hamilton-Jacobi form. This is generalized by the presence of a new term which takes into account the quantum effects via the quantum 
potential contribution

$$
Q(\boldsymbol{r}, t)=-\left(\frac{\hbar^{2}}{2 \mu}\right) \frac{\nabla^{2} R(\boldsymbol{r}, t)}{R(\boldsymbol{r}, t)} .
$$

$S$ is the SQM version of the Hamilton principal function. The equations of motion are computed from

$$
\begin{gathered}
\dot{\boldsymbol{r}}(t)=\boldsymbol{v}(\boldsymbol{r}(t), t), \\
\boldsymbol{v}(\boldsymbol{r}, t)=\frac{1}{\mu} \nabla S(\boldsymbol{r}, t)=\frac{\hbar}{\mu} \operatorname{Im}\left(\psi^{-1} \nabla \psi\right)
\end{gathered}
$$

here $\boldsymbol{v}(\boldsymbol{r}, t)$ is the velocity of the particle that passes through the point $\boldsymbol{r}$ at time $t$.

Eq. (5b) highlights the statistical character of the theory and is interpreted as a continuity equation. As with the classical statistical description, one introduces quantities which account for the particle properties of an ensemble of identical systems (same Hamiltonian, same quantum state, etc.) with trajectories. If the initial distribution in configuration space $\rho\left(\boldsymbol{r}, t_{0}\right)$ is assumed to be given by $\rho\left(\boldsymbol{r}, t_{0}\right)=R^{2}\left(\boldsymbol{r}, t_{0} 0\right)=\left|\psi\left(\boldsymbol{r}, t_{0}\right)\right|^{2}$ then the distribution $\rho(\boldsymbol{r}, t)$ satisfies the continuity equation provided that $\rho(\boldsymbol{r}, t)=|\psi(\boldsymbol{r}, t)|^{2}$ at all times. This expresses the time invariance of the configuration-space measure " $\rho(\boldsymbol{r}, t) d^{3} \boldsymbol{r}$ ". The probability distribution in configuration space given by $\rho=|\psi|^{2}$ is called the quantum equilibrium distribution. A system is then said to be in quantum equilibrium when its configurations are randomly distributed according to the quantum equilibrium distribution [4]. This is the so-called quantum equilibrium hypotesis (QEH): if a system is described by the wave function $\psi$ then its configurations are distributed according to $\rho=|\psi|^{2}$.

The fact that $R^{2}(\boldsymbol{r}, t)=|\psi(\boldsymbol{r}, t)|^{2}$ is the probability density that the particle is at $\boldsymbol{r}$ at time $t$ holds true in SQM, assures that one finds the same results as in QM. The probability density $|\psi(\boldsymbol{r}, 0)|^{2}$ gives information on the initial conditions necessary for the quantum HamiltonJacobi theory to be applied, thereby allowing the determination of particle trajectories through Eqs.(17).

In SQM, the probability density at time $t$ is related to particle trajectories. Closely analogous to classical statistical mechanics, starting from the initial distribution $\rho\left(\boldsymbol{r}_{0}, t_{0}\right)=$ $\left|\psi\left(\boldsymbol{r}_{0}, t_{0}\right)\right|^{2}$, one has:

$$
\rho(\boldsymbol{r}, t)=\int d^{3} \boldsymbol{r}_{0} \delta\left[\boldsymbol{r}_{0}-\boldsymbol{r}\left(t, \boldsymbol{r}_{0}\right)\right] \rho\left(\boldsymbol{r}_{0}, 0\right)=|\psi(\boldsymbol{r}, t)|^{2}
$$

Averaging the initial position $\boldsymbol{r}_{0}$ then yields the same average values of the corresponding operators in quantum mechanics. 
Consider the Hermitian operator $\hat{A}=\hat{A}(\hat{\boldsymbol{r}}, \hat{\boldsymbol{p}})$. In the $\boldsymbol{r}$ representation, in a state $\psi(\boldsymbol{r}, t)=$ $\langle\boldsymbol{r} \mid \psi(t)\rangle$, one has

$$
\langle\hat{A}\rangle_{t}=\int \psi^{*}(\boldsymbol{r}, t) \hat{A}(\boldsymbol{r},-i \hbar \nabla) \psi(\boldsymbol{r}, t) d^{3} \boldsymbol{r}
$$

That $\hat{A}$ is Hermitian, allows the definition of a local expectation value which, when integrated over all space, yields the average value $\langle\hat{A}\rangle$. One defines

$$
\mathcal{A}(\boldsymbol{r}, t)=\Re e\left(\frac{\psi^{*}(\boldsymbol{r}, t) \hat{A} \psi(\boldsymbol{r}, t)}{\psi^{*}(\boldsymbol{r}, t) \psi(\boldsymbol{r}, t)}\right),
$$

such that

$$
\langle\hat{A}\rangle_{t}=\int \mathcal{A}(\boldsymbol{r}, t) R^{2}(\boldsymbol{r}, t) d^{3} \boldsymbol{r}
$$

\section{TWO-TIME CORRELATION FUNCTIONS}

In the previous section it was shown how the two theories (SQM and QM) are completely equivalent if one considers average values of operators at one fixed time. As already anticipated above, in order to distinguish the two theories, one needs to consider two-time dependent quantities such as the correlation functions defined in Eq.(11). The averaging procedure is specified in what follows for both QM and SQM.

\section{A. Stochastic Quantum Mechanics}

As discussed in the previous section, the Bohm approach is able to reproduce the density distribution at time $t$ from the initial probability distribution $\rho\left(\boldsymbol{r}_{0}, t_{0}\right)=\left|\psi\left(\boldsymbol{r}_{0}, t_{0}\right)\right|^{2}$ at time $t_{0}$ while still employing the notion of trajectories (see Eq. (91)). For the time evolution of a general quantity $\mathcal{A}$, the quantum equilibrium hypothesis (QEH) gives (see Eqs.(9] and 12):

$$
\langle\hat{A}\rangle_{t}=\int \rho(\boldsymbol{r}, t) \mathcal{A}(\boldsymbol{r}, t) d^{3} \boldsymbol{r}=\int \rho\left(\boldsymbol{r}_{0}, 0\right) \mathcal{A}\left(\boldsymbol{r}\left(\boldsymbol{r}_{0}, t\right), 0\right) d^{3} \boldsymbol{r}_{0}
$$

The average value of two operators depending on different times is a generalization of Eq.(13). Given any two dynamic variables, $\mathcal{A}(\boldsymbol{r}, t)$ and $\mathcal{B}(\boldsymbol{r}, t)$, the average value of their product, weighed over the initial condition by the weight function $\left|\psi\left(\boldsymbol{r}_{0}, 0\right)\right|^{2}=\rho\left(\boldsymbol{r}_{0}\right)$, is just the SQM two-time correlation function[13]; It is

$$
\Phi^{A B}\left(t-t^{\prime}\right)=\int \rho\left(\boldsymbol{r}_{0}\right) \mathcal{A}(\boldsymbol{r}, t) \mathcal{B}\left(\boldsymbol{r}, t^{\prime}\right) d^{3} \boldsymbol{r}_{0}
$$

where $\mathcal{B}\left(\boldsymbol{r}, t^{\prime}\right)=\mathcal{B}\left(\boldsymbol{r}\left(t^{\prime}, \mathbf{r}_{0}\right), 0\right)$ and similarly $\mathcal{A}(\boldsymbol{r}, t)=\mathcal{A}\left(\boldsymbol{r}\left(t, \boldsymbol{r}_{0}\right), 0\right)$. 


\section{B. Quantum Theory}

To simplify the notation, consider the case of a time independent Hamiltonian with a discrete spectrum of eigenvalues

$$
H|N\rangle=E_{N}|N\rangle .
$$

In QM the two-time correlation function for a given state $|N\rangle$ is

$$
\Phi_{N}^{A B}\left(t-t^{\prime}\right)=\frac{1}{2}\left\langle N\left|\hat{A}(t) \hat{B}\left(t^{\prime}\right)+\hat{B}\left(t^{\prime}\right) \hat{A}(t)\right| N\right\rangle,
$$

where (in the Heisenberg representation)

$$
\hat{A}(t)=e^{i \hat{H} t / \hbar} \hat{A} e^{-i \hat{H} t / \hbar} .
$$

Consider the special case $\hat{A}=\hat{B}$. Then Eq. (16) reduces to

$$
\Phi_{N}^{A A}\left(t-t^{\prime}\right)=\frac{1}{2}\left(\Phi_{N+}^{A A}\left(t-t^{\prime}\right)+\Phi_{N-}^{A A}\left(t-t^{\prime}\right)\right),
$$

where

$$
\Phi_{N \pm}^{A A}\left(t-t^{\prime}\right)=\frac{1}{2} \sum_{M}|\langle M|\hat{A}| N\rangle|^{2} e^{\mp i \omega_{M N}\left(t-t^{\prime}\right)}
$$

and $\hbar \omega_{M N}=\left(E_{M}-E_{N}\right)$. The Hamiltonian being time independent is reflected by the fact that the $\Phi_{N}^{A, B}$ depend just on $\left(t-t^{\prime}\right)$.

The related noise spectral functions, defined as in Eq.(21) in QM are easily found to be given by

$$
S_{N}(\omega)=S_{N}^{+}(\omega)+S_{N}^{-}(\omega),
$$

with

$$
S_{N}^{ \pm}(\omega)=\frac{1}{2} \sum_{M}|\langle M|\hat{A}| N\rangle|^{2} \delta\left(\omega \mp \omega_{M N}\right) .
$$

When $|N\rangle$ is the ground state only $S_{N}^{+}$contributes for $\omega \geq 0$. In general, $S_{N}(\omega)$ is an even function of $\omega$. Furthermore, if the Hamiltonian has a spectrum with both a discrete and a continuous part, then the sums in the above Eqs.(20121) clearly split into a corresponding sum over the discrete part of the spectrum plus an integral over the continuous one. It will prove useful in deriving the asymptotic behavior of the QM noise spectral function $S_{N}(\omega)$ (in the next section) to relate it to the Fourier transform of the average of the time-ordered product; i.e.

$$
\alpha_{N}(\omega)=\frac{i}{\hbar} \int_{-\infty}^{+\infty} e^{i \omega t}\langle N|T[\hat{A}(t) \hat{A}(0)]| N\rangle d t
$$


where "T" denotes operator time ordering. The quantities $S_{N}(\omega)$ and $\alpha_{N}(\omega)$ are related by

$$
S_{N}(\omega)=\frac{\hbar}{2 \pi} \operatorname{Im} \alpha_{N}(\omega)
$$

\section{THE ELECTRIC DIPOLE MOMENT AND THE HYDROGEN ATOM}

\section{A. Stochastic Quantum Mechanics}

Consider the electric dipole moment along the $x$ axis $d_{x}(t)=e x(t)$. In the Bohm description, the two-time correlation function of the electric dipole moment for a generic excited eigenstate of the hydrogen atom with quantum numbers $(n, l, m)$ is as given in Eq. (14); i.e.

$$
\begin{aligned}
\Phi_{n l m}\left(t, t^{\prime}\right) & =\left\langle d_{x}(t) d_{x}\left(t^{\prime}\right)\right\rangle_{n l m} \\
& =\int d_{x}\left(\boldsymbol{r}\left(t, \boldsymbol{r}_{0}\right)\right) d_{x}\left(\boldsymbol{r}\left(t^{\prime}, \boldsymbol{r}_{0}\right)\right) \rho_{n l m}\left(\boldsymbol{r}_{0}\right) d^{3} \boldsymbol{r}_{0}
\end{aligned}
$$

where $d_{x}\left(\boldsymbol{r}\left(t, \boldsymbol{r}_{0}\right)\right)=e r_{0} \sin \theta_{0} \cos \phi(t)$. Using the hydrogen atom bound state wave functions and the solution to the equations of motion in the Bohm description, given in appendix A, it is straightforward to derive the following properties of the related noise spectral functions

$$
\begin{aligned}
S_{n, l, m}(\omega) & =S_{n, l, m}(-\omega), \\
S_{n, l, m}(\omega) & =S_{n, l,-m}(\omega), \\
S_{n, l, 0}(\omega) & \propto \delta(\omega) .
\end{aligned}
$$

Thus, without loss of generality, $m>0$ and $\omega>0$ can be assumed in the following discussion. For general quantum numbers, one can derive:

$$
S_{n l m}(\omega)=\frac{c_{n m l}}{128}\left(\frac{e^{2} a_{0}^{2}}{\omega_{0}}\right)\left[z_{n, m}(\omega)\right]^{2(3+m)} \int_{z_{n, m}(\omega)}^{\infty} e^{-\rho} \rho^{2(l-m)}\left[L_{n+l}^{2 l+1}(\rho)\right]^{2}\left(\frac{\left[C_{l-m}^{(m+1 / 2)}(\xi)\right]^{2}}{\xi}\right) d \rho,
$$

where

$$
\begin{aligned}
\omega_{0} & =\left(\hbar / \mu a_{0}^{2}\right) \\
z_{n, m}(\omega) & =\frac{2}{n} \sqrt{\frac{m \omega_{0}}{\omega}} \\
\xi & =\sqrt{1-\left(\frac{z_{n, m}(\omega)}{\rho}\right)^{2}}
\end{aligned}
$$




$$
c_{n l m}=\left[\frac{n^{4}(2 l+1)(l-m) !((2 m-1) ! !)^{2}(n-l-1) !}{2 n m(l+m) ![(n+l) !]^{3}}\right],
$$

and $C_{l-m}^{(m+1 / 2)}(\xi)$ are the ultra-spherical Gegenbauer polynomials [14] which satisfy the following relations:

$$
\begin{aligned}
C_{l-m}^{(m+1 / 2)}(1) & =\frac{(l+m) !}{(2 m) !(l-m) !} \\
C_{0}^{(m+1 / 2)}(\xi) & =1 \\
\frac{C_{l-m}^{(m+1 / 2)}(\xi)}{1 \cdot 3 \cdots(2 m-1)} & =\left(\frac{d}{d \xi}\right)^{m} P_{l}(\xi),
\end{aligned}
$$

where $P_{l}(\xi)$ is the Legendre polynomial.

A few special cases have been explicitly computed and are here reported using $\mathcal{S}_{n l m}(\omega)$ to denote the noise spectral function of Eq.(26) in units of $\left(e^{2} a_{0}^{2}\right) / \omega_{0}$ :

$$
S_{n l m}^{\mathrm{SQM}}(\omega)=\left(\frac{e^{2} a_{0}^{2}}{\omega_{0}}\right) \mathcal{S}_{n l m}(\omega)
$$

$\underline{n=2}$

$$
\mathcal{S}_{211}(\omega)=\frac{1}{128}\left(\frac{\omega_{0}}{\omega}\right)^{4} z_{2,1} K_{1}\left(z_{2,1}\right)
$$

$\underline{n=3}$

$$
\begin{aligned}
& \mathcal{S}_{322}(\omega)=\left(\frac{1}{2187}\right)\left(\frac{\omega_{0}}{\omega}\right)^{5} z_{3,2} K_{1}\left(z_{3,2}\right) \\
& \mathcal{S}_{321}(\omega)=\left(\frac{1}{3888}\right)\left(\frac{\omega_{0}}{\omega}\right)^{4} z_{3,1}\left[2 K_{1}\left(z_{3,1}\right)+z_{3,1} K_{0}\left(z_{3,1}\right)\right] \\
& \mathcal{S}_{311}(\omega)=\left(\frac{1}{243}\right)\left(\frac{\omega_{0}}{\omega}\right)^{4} z_{3,1}\left[\left(\frac{5}{8}+\frac{z_{3,1}^{2}}{16}\right) K_{1}\left(z_{3,1}\right)-\frac{7}{16} z_{3,1} K_{0}\left(z_{3,1}\right)\right]
\end{aligned}
$$

$\underline{n} \quad(l=m=n-1)$

$$
\begin{aligned}
\mathcal{S}_{n, n-1, n-1}(\omega) & =c_{n}\left(\frac{\omega_{0}}{\omega}\right)^{n+2} z_{n, n-1} K_{1}\left(z_{n, n-1}\right) \\
c_{n} & =\frac{1}{8}\left(\frac{2}{n}\right)^{2 n}(n-1)^{n+1} \frac{[(2 n-3) ! !]^{2}}{2 n[(2 n-2) !]^{2}}
\end{aligned}
$$

$n \quad(l=n-1, m=n-2)$

$$
\begin{aligned}
\mathcal{S}_{n, n-1, n-2}(\omega) & =\bar{c}_{n}\left(\frac{\omega_{0}}{\omega}\right)^{n+1} z_{n, n-2}^{2} K_{2}\left(z_{n, n-2}\right) \\
\bar{c}_{n} & =c_{n, n-1, n-2}[(2 n-1) !(2 n-3) ! !]^{2} \frac{4(n-2)}{128 n^{2}}
\end{aligned}
$$


In the above expressions, $K_{n}(z)$ are the $n^{t h}$ order modified Bessel functions. It should be noted that the function $S_{211}(\omega)$ agrees exactly with the numerical computation previously reported [12]. It has been verified that the particular results of Eqs.(30) and (311) are consistent with general formulas given in Eqs. (32) and (33). In Fig.1 we show the plots of some of the above explicit examples.

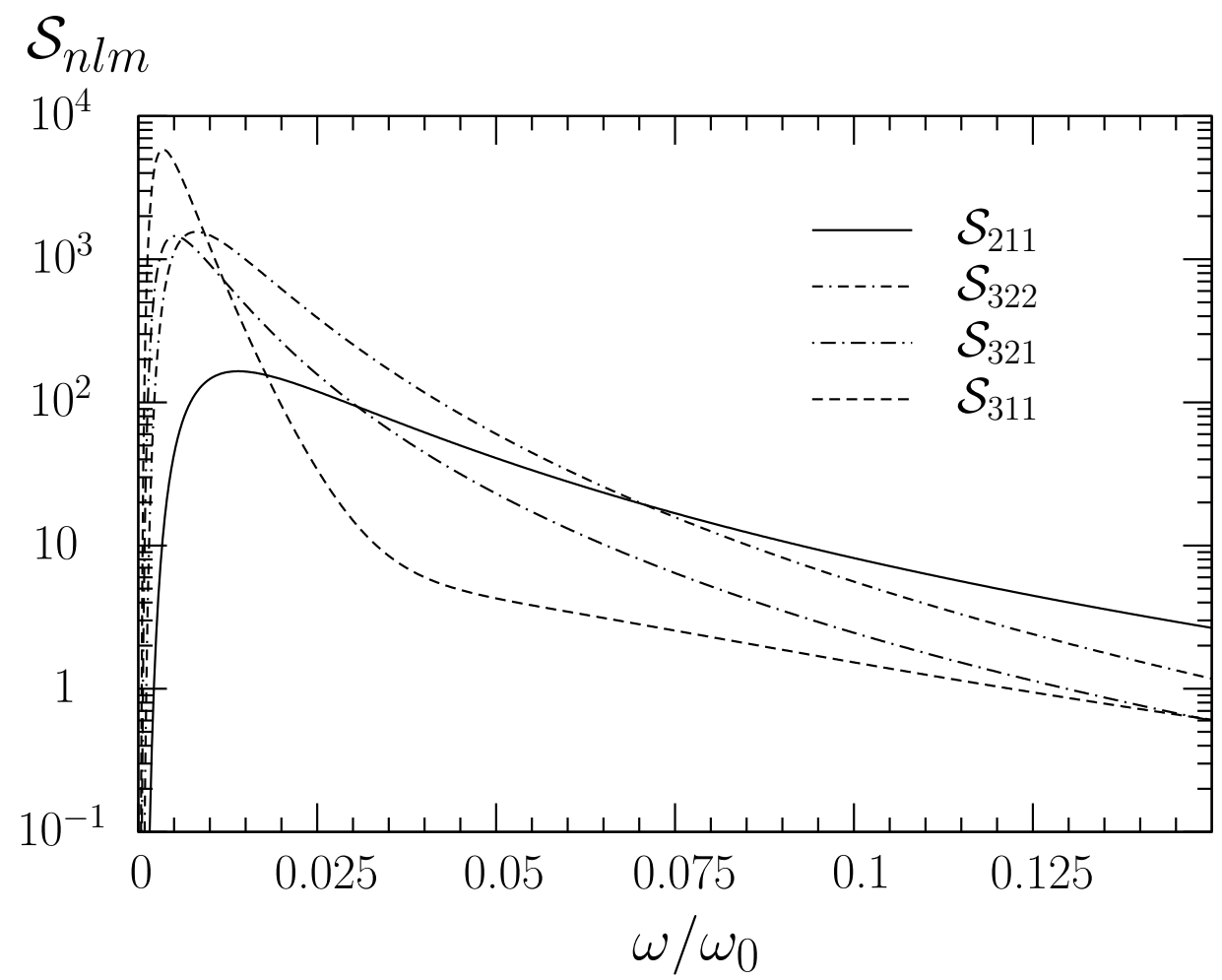

FIG. 1: Shown are four plots of typical spectral functions $\mathcal{S}_{n l m}(\omega)=\omega_{0} S_{n l m}^{\mathrm{SQM}}(\omega) /\left(e a_{0}\right)^{2}$ for the electric dipole moment of the Hydrogen atom computed employing the Stochastic Quantum Mechanics.

\section{Asymptotic behavior.}

From Eq.(26) it is possible to derive for general quantum numbers the asymptotic behavior at large frequencies of the SQM noise spectral function; it is

$$
S_{n l m}^{\mathrm{SQM}}(\omega) \rightarrow \mathcal{C}_{n l m}\left(\frac{e^{2} a_{0}^{2}}{\omega_{0}}\right)\left(\frac{\omega_{0}}{\omega}\right)^{3+m} \text { as } \omega \rightarrow \infty,
$$

where

$$
\mathcal{C}_{n l m}=\frac{c_{n l m}}{128}\left[C_{l-m}^{(m+1 / 2)}(1)\right]^{2}\left(\frac{4 m}{n^{2}}\right)^{3+m} \int_{0}^{\infty} e^{-\rho} \rho^{2(l-m)}\left[L_{n+l}^{(2 l+1)}(\rho)\right]^{2} d \rho .
$$


Thus, noise spectral functions vanish as $\omega \rightarrow \infty$ with a power law. The exponent is related to the state's quantum numbers by $(3+m)$. The explicit cases considered above can easily be shown to agree with Eq.(34). One may use the expansion of the modified Bessel functions for small values of the argument [14], i.e. as $z \rightarrow 0$

$$
K_{\nu}(z) \rightarrow \begin{cases}(1 / 2) \Gamma(\nu)(z / 2)^{-\nu} & \text { if } \nu \neq 0 \\ -\ln (z) & \text { if } \nu=0\end{cases}
$$

One then infers the large $\omega \rightarrow \infty$ behavior of the spectral functions; i.e.

$$
S_{n l m}^{\mathrm{SQM}}(\omega) \rightarrow \begin{cases}(1 / 128)\left(\frac{\omega_{0}}{\omega}\right)^{4} & \text { for state }|211\rangle, \\ (1 / 2187)\left(\frac{\omega_{0}}{\omega}\right)^{5} & \text { for state }|322\rangle \\ (1 / 1944)\left(\frac{\omega_{0}}{\omega}\right)^{4} & \text { for state }|321\rangle \\ (5 / 1944)\left(\frac{\omega_{0}}{\omega}\right)^{4} & \text { for state }|311\rangle\end{cases}
$$

\section{B. Quantum Mechanics}

As anticipated at the end of Sec.III, in order to derive the asymptotic behavior of the QM noise spectral function it proves useful to work with the "time ordered propagator" defined in Eq.(22) and use Eq.(23) to find $S_{N}(\omega)$. The function $\alpha_{N}(\omega)$ can easily be connected with the (retarded) Green's function of the Schrödinger equation; i.e.

$$
G_{r e t}\left(\boldsymbol{r}_{2}, \boldsymbol{r}_{1} ; \omega\right)=-\frac{i}{\hbar} \int_{0}^{\infty}\left\langle\boldsymbol{r}_{2}\left|e^{i\{\omega-(H / \hbar)\} t}\right| \mathbf{r}_{1}\right\rangle d t
$$

The time ordered response function $\alpha_{N}^{(A B)}(\omega)$ is related to the Green's function $G_{r e t}\left(\boldsymbol{r}_{2}, \boldsymbol{r}_{1} ; \omega\right)$ via

$$
\begin{aligned}
-\alpha_{N}^{(A B)}(\omega)= & \int d^{3} \boldsymbol{r}_{1} \int d^{3} \boldsymbol{r}_{2}\left\langle N|A(0)| \boldsymbol{r}_{2}\right\rangle G_{r e t}\left(\boldsymbol{r}_{2}, \boldsymbol{r}_{1} ; \frac{E_{N}}{\hbar}+\omega\right)\left\langle\boldsymbol{r}_{1}|B(0)| N\right\rangle+ \\
& \int d^{3} \boldsymbol{r}_{1} \int d^{3} \boldsymbol{r}_{2}\left\langle N|B(0)| \boldsymbol{r}_{2}\right\rangle G_{r e t}\left(\boldsymbol{r}_{2}, \boldsymbol{r}_{1} ; \frac{E_{N}}{\hbar}-\omega\right)\left\langle\boldsymbol{r}_{1}|A(0)| N\right\rangle
\end{aligned}
$$

The retarded Green's function associated to the Hamiltonian H, as defined in Eq.(38), satisfies the differential equation

$$
\left(\hbar \omega-H_{\left(\boldsymbol{r}_{2}\right)}\right) G_{r e t}\left(\boldsymbol{r}_{2}, \boldsymbol{r}_{1} ; \omega\right)=\delta\left(\boldsymbol{r}_{2}-\boldsymbol{r}_{1}\right),
$$


where $H_{\left(\boldsymbol{r}_{2}\right)}=-\left(\hbar^{2} / 2 \mu\right) \nabla_{\left(\boldsymbol{r}_{2}\right)}^{2}-\left(Z e^{2} / r_{2}\right)$ denotes a one electron Coulomb system. The differential equation of the non-relativistic Coulomb Green's function (in the standard normalization) is [15, 16, 17]

$$
\left\{\nabla_{\left(\boldsymbol{r}_{2}\right)}^{2}+\left(\frac{2 k \nu}{r_{2}}\right)+k^{2}\right\} G\left(\boldsymbol{r}_{2}, \boldsymbol{r}_{1} ; \omega\right)=\delta\left(\boldsymbol{r}_{2}-\boldsymbol{r}_{1}\right)
$$

where $k=\sqrt{(2 \mu \omega / \hbar)}$ and $\nu=4 \pi \hbar^{2} /\left(Z \mu e^{2} k\right)$.

The Green's functions appearing in Eqs.(38) and (41) are related by a normalization constant $G_{r e t}\left(\boldsymbol{r}_{2}, \boldsymbol{r}_{1} ; \omega\right)=\left(2 \mu / \hbar^{2}\right) G\left(\boldsymbol{r}_{2}, \boldsymbol{r}_{1} ; \omega\right)$. A closed expression of the Coulomb Green's function in terms of Whittaker functions [14] has been given by L. Hostler [15] as

$$
G\left(\boldsymbol{r}_{2}, \boldsymbol{r}_{1} ; \omega\right)=-\frac{\Gamma(1-i \nu)}{4 \pi\left|\boldsymbol{r}_{2}-\boldsymbol{r}_{1}\right|} \operatorname{det}\left(\begin{array}{ll}
\mathcal{W}_{i \nu ; 1 / 2}\left(-i k \alpha_{2}\right) & \mathcal{M}_{i \nu ; 1 / 2}\left(-i k \alpha_{1}\right) \\
\dot{\mathcal{W}}_{i \nu ; 1 / 2}\left(-i k \alpha_{1}\right) & \dot{\mathcal{M}}_{i \nu ; 1 / 2}\left(-i k \alpha_{2}\right)
\end{array}\right)
$$

where the dots over the Whittaker functions denote differentiation with respect to their arguments and

$$
\begin{aligned}
& \alpha_{2}=r_{2}+r_{1}+\left|\boldsymbol{r}_{2}-\boldsymbol{r}_{1}\right| \\
& \alpha_{1}=r_{2}+r_{1}-\left|\boldsymbol{r}_{2}-\boldsymbol{r}_{1}\right| .
\end{aligned}
$$

\section{Asymptotic behavior.}

The aim of this section is to derive the asymptotic form of the noise spectral functions as $\omega \rightarrow \infty$. Using Eq.(39) requires the Coulomb Green's function in the regime $\omega \rightarrow \infty$ (respectively $\omega \rightarrow-\infty$ ). In this limit, $k \rightarrow \infty$ (respectively $k \rightarrow i \infty$ ). Also $\nu \rightarrow 0$ so that Eq.(41) reduces to the differential equation of the free particle Green's function $G_{0}\left(\boldsymbol{r}_{2}, \boldsymbol{r}_{1} ; \omega\right)$. Indeed, from the exact solution in Eq.(42), it is possible to show explicitly that as $|\omega| \rightarrow \infty$

$$
G\left(\boldsymbol{r}_{2}, \boldsymbol{r}_{1} ; \omega\right) \rightarrow G_{0}\left(\boldsymbol{r}_{2}, \mathbf{r}_{1} ; \omega\right)=-\frac{e^{i k\left|\boldsymbol{r}_{2}-\boldsymbol{r}_{1}\right|}}{4 \pi\left|\boldsymbol{r}_{2}-\boldsymbol{r}_{1}\right|}
$$

This proves that the exact Coulomb Green's function has an oscillatory behavior at large positive frequencies and an exponentially damped behavior at large negative frequencies:

$$
\begin{aligned}
& -4 \pi\left|\boldsymbol{r}_{2}-\boldsymbol{r}_{1}\right| G \rightarrow e^{i\left|\boldsymbol{r}_{2}-\mathbf{r}_{1}\right| \sqrt{2 \mu \omega / \hbar}} \quad \text { as } \omega \rightarrow+\infty \\
& -4 \pi\left|\boldsymbol{r}_{2}-\boldsymbol{r}_{1}\right| G \rightarrow e^{-\left|\boldsymbol{r}_{2}-\mathbf{r}_{1}\right| \sqrt{2 \mu|\omega| / \hbar}} \text { as } \omega \rightarrow-\infty
\end{aligned}
$$

Inserting this result into the central Eq.(39), one finds that only the first term survives; i.e. for $\omega \rightarrow \infty$

$$
\left(\hbar^{2} / 2 \pi \mu\right) \alpha_{N}^{(A B)}(\omega) \rightarrow \int d^{3} \boldsymbol{r}_{1} \int d^{3} \boldsymbol{r}_{2}\left\langle N|A(0)| \boldsymbol{r}_{2}\right\rangle \frac{e^{i k\left|\boldsymbol{r}_{2}-\boldsymbol{r}_{1}\right|}}{\left|\boldsymbol{r}_{2}-\boldsymbol{r}_{1}\right|}\left\langle\mathbf{r}_{2}|B(0)| N\right\rangle .
$$


where it is to be recalled that $k=\sqrt{(2 \mu \omega / \hbar)}$.

The above considerations can be readily applied to the case of the electric dipole moment in the hydrogen atom with $A(0)=B(0)=e x$. As $\omega \rightarrow \infty$

$$
\alpha_{N}^{(x x)}(\omega) \rightarrow \frac{\mu e^{2}}{2 \pi \hbar^{2}} \int d^{3} \boldsymbol{r}_{1} d^{3} \boldsymbol{r}_{2} \psi_{N}^{*}\left(\boldsymbol{r}_{2}\right) x_{2} \frac{e^{i k\left|\boldsymbol{r}_{2}-\boldsymbol{r}_{1}\right|}}{\left|\boldsymbol{r}_{2}-\mathbf{r}_{1}\right|} x_{1} \psi_{N}\left(\boldsymbol{r}_{1}\right)
$$

Using the above in Eq.(23), the asymptotic expression for the electric dipole moment spectral function is derived; i.e.

$$
S_{N}^{(x x)}(\omega) \rightarrow \frac{e^{2}}{4 \pi^{2} a_{0}^{2} \omega_{0}} \operatorname{Im} \int d^{3} \boldsymbol{r}_{1} \int d^{3} \boldsymbol{r}_{2} \psi_{N}^{*}\left(\boldsymbol{r}_{2}\right) x_{2} \frac{e^{i k\left|\boldsymbol{r}_{2}-\boldsymbol{r}_{1}\right|}}{\left|\boldsymbol{r}_{2}-\boldsymbol{r}_{1}\right|} x_{1} \psi_{N}\left(\boldsymbol{r}_{1}\right) .
$$

With $|N\rangle=|n l m\rangle$, the hydrogen atom wave functions are written as $\psi_{n l m}(\boldsymbol{r})=$ $\chi_{n l}(r) Y_{l m}(\theta, \phi)$. One may also employ the expansion

$$
\frac{e^{i k\left|\boldsymbol{r}_{2}-\boldsymbol{r}_{1}\right|}}{\left|\boldsymbol{r}_{2}-\boldsymbol{r}_{1}\right|}=(4 \pi i k) \sum_{l=0}^{\infty} j_{l}\left(k r_{<}\right) h_{l}^{(1)}\left(k r_{>}\right) \sum_{m=-l}^{l} Y_{l m}^{*}\left(\theta_{1}, \phi_{1}\right) Y_{l m}\left(\theta_{2}, \phi_{2}\right),
$$

where $r_{<}=\min \left(r_{1}, r_{2}\right)$ and $r_{>}=\max \left(r_{1}, r_{2}\right)$. The noise spectral function then has the asymptotic limit

$$
S_{n l m}^{(x x)}(\omega) \rightarrow \frac{e^{2}}{\pi \omega_{0} a_{0}^{2}} \operatorname{Im}\left\{i k \sum_{l^{\prime}=0}^{\infty} C_{l m}^{l^{\prime}} \int_{0}^{\infty} \int_{0}^{\infty} d r_{1} d r_{2}\left(r_{2} r_{1}\right)^{3} \chi_{n l}\left(r_{2}\right) j_{l^{\prime}}\left(k r_{<}\right) h_{l^{\prime}}^{(1)}\left(k r_{>}\right) \chi_{n l}\left(r_{1}\right)\right\},
$$

with the constants $C_{l m}^{\left(l^{\prime}\right)}$ defined as

$$
C_{l m}^{\left(l^{\prime}\right)}=\sum_{m^{\prime}-l^{\prime}}^{+l^{\prime}}\left|\int Y_{l^{\prime} m^{\prime}}^{*}(\theta, \phi) \sin \theta \cos \phi Y_{l m}(\theta, \phi) d \Omega\right|^{2}
$$

When taking the imaginary part in Eq.(50), only the function $h_{l^{\prime}}^{(1)}(k r)$ is complex[14];

$$
\operatorname{Im}\left\{i h_{l^{\prime}}^{(1)}(k r)\right\} \rightarrow j_{l}(k r)=\sqrt{\pi /(2 k r)} J_{l+1 / 2}(k r) .
$$

Therefore, the noise spectral function as $\omega \rightarrow \infty$ reads

$$
S_{n l m}^{(x x)}(\omega) \rightarrow\left(\frac{e^{2}}{2 \omega_{0} a_{0}^{2}}\right) \sum_{l^{\prime}=0}^{\infty} C_{l m}^{\left(l^{\prime}\right)}\left|\int_{0}^{\infty} r^{5 / 2} \chi_{n l}(r) J_{l^{\prime}+1 / 2}(k r) d r\right|^{2} .
$$

The radial integral in the above expression can be evaluated in the limit of high frequencies and is found to vanish as $\left(k a_{0}\right)^{-(4+l+1 / 2)}$. Indeed, using the hydrogen wave functions reported 
in the appendix, one finds

$$
\begin{aligned}
I_{n l l^{\prime}} & =\int_{0}^{\infty} \sqrt{r}\left(\frac{r}{a_{0}}\right)^{2} \chi_{n l}(r) J_{l^{\prime}+1 / 2}(k r) d r \\
& =-\frac{2}{n^{2}}\left(\frac{(n-l-1) !}{[(n+l) !]^{3}}\right)^{1 / 2} \frac{1}{\left(k a_{0}\right)^{(4+l-1 / 2)}}\left(\frac{2}{n}\right)^{l} \int_{0}^{\infty} d x x^{(3+l-1 / 2)} e^{-\left(x / n k a_{0}\right)} \times \\
& L_{n+l}^{2 l+1}\left(2 x / n k a_{0}\right) J_{l^{\prime}+1 / 2}(x)
\end{aligned}
$$

When $k \rightarrow \infty$ the Laguerre polynomial can be replaced by the constant value that it takes for a vanishing argument $L_{n+l}^{2 l+1}(0)$. The remaining integral is tabulated (see Eq.(6.621) in [18]) and one finds

$$
\begin{aligned}
I_{n l l^{\prime}} \approx-\frac{2}{n^{2}}\left(\frac{(n-l-1) !}{[(n+l) !]^{3}}\right)^{1 / 2} \frac{1}{\left(k a_{0}\right)^{(4+l-1 / 2)}}\left(\frac{2}{n}\right)^{l} L_{n+l}^{2 l+1}(0) \Gamma\left(l+l^{\prime}+4\right) \times \\
\\
\left\{P_{3+l-1 / 2}^{-\left(l^{\prime}+1 / 2\right)}(0)+\frac{1}{n k a_{0}}\left[\frac{d}{d x} P_{3+l-1 / 2}^{-\left(l^{\prime}+1 / 2\right)}(0)\right]\right\}
\end{aligned}
$$

where $P_{\mu}^{\nu}(x)$ are the associated Legendre functions of the first kind. One should note that for the sum in Eq.(53) only few terms are non-vanishing. This is related to well known electric dipole selection rules which apply when calculating the quantities

$$
C_{l m}^{\left(l^{\prime}\right)}=\sum_{m^{\prime}=-l^{\prime}}^{l^{\prime}}\left|\left\langle l^{\prime} m^{\prime}|\sin \theta \cos \phi| l m\right\rangle\right|^{2}
$$

i.e.

$$
\left\langle l^{\prime} m^{\prime}|\sin \theta \cos \phi| l m\right\rangle \neq 0 \text { only if }\left\{\begin{array}{l}
m^{\prime}=m \pm 1 \\
l^{\prime}=l \pm 1
\end{array}\right.
$$

Applying the selection rule, $l^{\prime}=l \pm 1$, it turns out that $P_{3+l-1 / 2}^{-\left(l^{\prime}+1 / 2\right)}(0)$ vanishes for any $l$, while its first derivative at zero is always finite (see Eqs.(8.6.1) and (8.6.3) in [14]). This completes the proof of the asymptotic behavior of the noise spectral functions in QM. Taking into account that $k a_{0}=\sqrt{2 \omega / \omega_{0}}$, one finally concludes that for $\omega \rightarrow \infty$,

$$
S_{n l m}^{\mathrm{QM}}(\omega) \rightarrow\left(\frac{e^{2} a_{0}^{2}}{\omega_{0}}\right) \mathcal{C}_{n l m}^{\prime}\left(\frac{\omega_{0}}{\omega}\right)^{4+l+1 / 2}
$$

where

$$
\mathcal{C}_{n l m}^{\prime}=\frac{2}{n^{4}} \sum_{l^{\prime}=l \pm 1} C_{l m}^{\left(l^{\prime}\right)} \frac{(n-l-1) !}{[(n+l) !]^{3}}\left[L_{n+l}^{2 l+1}(0)\right]^{2}\left\{\frac{\Gamma\left(l+l^{\prime}+4\right)}{n(\sqrt{2})^{4+l+1 / 2}}\left[\frac{d}{d x} P_{3+l-1 / 2}^{-\left(l^{\prime}+1 / 2\right)}(0)\right]\right\}^{2} .
$$

When comparing Eq.(57) with Eq.(34) a difference in the two descriptions, SQM and QM is made very clear. As $\omega \rightarrow \infty, \mathrm{SQM}$ predicts for noise spectral functions in state $|n l m\rangle$ to decrease at large frequencies as $\omega^{-(3+m)}$ and QM as $\omega^{-(4+l+1 / 2)}$. 


\section{SUM RULES: MOMENTS OF THE NOISE SPECTRAL FUNCTIONS}

In highlighting possible differences between the predictions of Quantum Mechanics and the Stochastic Quantum Mechanics, it proves useful to study some global properties of the spectral function, e.g. sum rules. The zeroth order moment is readily evaluated,

$$
\begin{aligned}
\gamma^{(0)} & =\int_{-\infty}^{+\infty} S^{A, A}(\omega) d \omega \\
& =\int_{-\infty}^{+\infty} \int_{-\infty}^{+\infty} e^{i \omega t} G^{A, A}(t) \frac{d \omega d t}{2 \pi} \\
& =G^{A, A}(0)=\left\langle A^{2}(0)\right\rangle .
\end{aligned}
$$

The zeroth moment $\gamma^{(0)}$ is nothing more than the average value of $\left\langle A^{2}\right\rangle$ at time zero and it is the same in QM and SQM. For the special case under consideration this assumes the value $\left\langle x^{2}(0)\right\rangle_{n l m}$.

$$
\gamma_{n l m}^{(0)}=\frac{a_{0}^{2}}{4} n^{2}\left[5 n^{2}+1-3 l(l+1)\right]\left[1-\frac{(l+m+1)(l-m+1)}{(2 l+1)(2 l+3)}-\frac{(l+m)(l-m)}{(2 l+1)(2 l-1)}\right] .
$$

On the contrary, the calculation of the second order moment $\gamma^{(2)}$ (again in both theories) shows the first discrepancy between QM and SQM. The computation of the second order moment can be related to the second derivative of the two-time correlation function calculated at $\tau=t-t^{\prime}=0$. Indeed repeated integration by parts yields

$$
\omega^{2} S_{N}(\omega)=-\int_{-\infty}^{+\infty} e^{i \omega \tau} \frac{d^{2} \Phi_{N}(\tau)}{d \tau^{2}}\left(\frac{d \tau}{2 \pi}\right)
$$

Thus

$$
\gamma_{N}^{(2)}=\int_{-\infty}^{+\infty} \omega^{2} S_{N}(\omega) d \omega=-\left(\frac{d^{2} \Phi_{N}(\tau)}{d \tau^{2}}\right)_{\tau=0}
$$

\section{A. Stochastic Quantum Mechanics}

In the Stochastic Quantum Mechanics from Eqs.(24) and (1A6) one has

$$
-\frac{d^{2} \Phi_{N}(\tau=0)}{d \tau^{2}}=\left(\frac{m \hbar e}{\mu}\right)^{2}\left\langle n l m\left|\frac{\cos ^{2} \phi}{r^{2} \sin ^{2} \theta}\right| n l m\right\rangle
$$

so that

$$
\gamma_{(\mathrm{SQM}) n l m}^{(2)}=\frac{e^{4}}{2 \mu a_{0}}\left(\frac{m}{n^{3}}\right)
$$


In QM one obtains

$$
-\frac{d^{2} \Phi_{n l m}(\tau=0)}{d \tau^{2}}=\frac{e^{2}}{2}\langle n l m|\{\ddot{x}(0), x(0)\}| n l m\rangle .
$$

Using the Coulomb Hamiltonian $H=\left(\boldsymbol{p}^{2} / 2 \mu\right)-\left(e^{2} / r\right)$ one may deduce the following commutators:

$$
\begin{aligned}
& \dot{x}=\frac{i}{\hbar}[H, x]=\frac{p_{x}}{\mu}, \\
& \ddot{x}=\frac{i}{\hbar}\left[H, p_{x}\right]=-\left(\frac{e^{2}}{\mu}\right) \frac{x}{r^{3}},
\end{aligned}
$$

so that

$$
-\frac{d^{2} \Phi_{n l m}(\tau=0)}{d \tau^{2}}=-\frac{e^{4}}{2 \mu}\left\langle n l m\left|\frac{x^{2}}{r^{3}}\right| n l m\right\rangle
$$

and

$$
\gamma_{(\mathrm{QM}) n l m}^{(2)}=\left(\frac{e^{4}}{\mu a_{0}} \frac{1}{n^{2}}\right)\left[1-\frac{(l+m+1)(l-m+1)}{(2 l+1)(2 l+3)}-\frac{(l+m)(l-m)}{(2 l+1)(2 l-1)}\right],
$$

which is quite different from the expression obtained in the Bohm theory Eq. (64). Clearly the differences found in the second order moment imply rigorously different spectral functions.

\section{B. Semi-classical limit}

It is interesting to see how the two quantities which are different for general quantum numbers have the same semi-classical limit for large values of the quantum numbers $n, l$ and $m$. Setting the maximum orbital momentum $l=m=n-1$ one finds that

$$
\begin{aligned}
\gamma_{(\mathrm{SQM}) n, n-1, n-1}^{(2)} & =\left(\frac{e^{4}}{2 a_{0} \mu}\right) \frac{(n-1)}{n^{3}}, \\
\gamma_{(\mathrm{QM}) n, n-1, n-1}^{(2)} & =\left(\frac{e^{4}}{2 a_{0} \mu}\right) \frac{1}{n(n+1 / 2)} .
\end{aligned}
$$

In the limit of large values of $n$ the two theories agree; i.e.

$$
\lim _{n \rightarrow \infty}\left\{n^{2} \gamma_{n, n-1, n-1}^{(2)}\right\}=\left(\frac{e^{4}}{2 a_{0} \mu}\right)
$$

for both QM and SQM as expected.

\section{CONCLUSIONS}

In this work the predictions of the Stochastic and conventinal Quantum Mechanics have been compared in some detail with respect to the two-time correlation functions. The 
example of interest in this work is the dynamic evolution of the electric dipole moment within the hydrogen atom. Previous numerical computations [12] of the SQM spectral function for the first excited state $|211\rangle$ have been confirmed. In addition closed expressions have been obtained in terms of modified Bessel functions $K_{n}(z)$, for several excited states with special combinations of quantum numbers. A derivation is provided of the asymptotic form (as $\omega \rightarrow \infty$ ) of the noise spectral functions for both QM and SQM. For large frequencies the two descriptions provide different power law behavior. For the hydrogen atom bound states $|n l m\rangle$ the SQM spectral functions scale $\propto \omega^{-(3+m)}$ as opposed to the QM spectral functions which scale $\propto \omega^{-(4+l+1 / 2)}$.

The difference in the noise spectral functions is reflected in different sum rules which are obeyed by the spectral functions. Explicit exact evaluations of the second order moment of the spectral functions $\gamma^{(2)}$, as in Eq.(3), show that the two descriptions predict indeed different values.

Finally by considering, for example, the interaction of an hydrogen atom with the field of an electromagnetic wave, it is possible to relate the noise spectral functions within an excited state to a total absorption cross-section by the relation $\sigma_{\text {tot }}^{N}(\omega)=8 \pi^{2} \alpha_{\mathrm{QED}}\left[\omega S_{N}(\omega)\right]$ as shown in detail in the appendix $\mathrm{C}$.

Let us briefly comment on the different predictions just so pointed out between conventional quantum mechanics and trajectory based interpretations of stochastic nature (Bhom or Nelson). It is certainly worthwhile to mention here a recent work 19] where the author shows that trajectory based interpretations of quantum mechanics are incomplete. This happens for systems with unbounded Hamiltonians. In particular it is shown that for particular systems (providing explicit examples) there exist states of finite energy for which the decomposition of the Schrödinger equation into a continuity and modified HamiltonJacobi equation is impossible. These examples are also shown to be connected to the fact that the corresponding state wave functions exhibit fractal properties. The main conclusion of ref. 19] is that Quantum Mechanics goes where trajectory interpretations do not follow despite their (in principle) duty to do so. So one might wonder whether the results found in the present work regarding two-time correlation functions are to be ascribed to such incompleteness of stochastic approaches to quantum mechanics. We can just remark that the states considered here to evidence differences in the predictions of the two theories are the bound states eigensolutions of the Hydrogen atom hamiltonian $\left(\left|\psi_{n l m}\right\rangle\right)$ and as such do not 
have the properties required in [19] to highlight the supposed incompleteness of Stochastic QM (i.e. undefined $H \psi$, but with finite average energy). It would certainly be interesting to consider the possibility to construct such states for the Hydrogen atom but this deserves further investigation, and goes beyond the scope of the present work. Were it possible to confirm this connection it would leave little doubt on the authors' minds as to which of the two theories would have to be ruled out.

\section{Acknowledgments}

The authors would like to thank the referee for bringing to their attention the interesting work of M. J. W Hall [19] about unbounded hamiltonians.

A.W. would like to thank the Dipartimento di Fisica, Università di Perugia and I.N.F.N. Sezione di Perugia for hospitality and support while this work was in progress.

\section{APPENDIX A: THE BOHM DESCRIPTION OF THE HYDROGEN ATOM}

A stationary eigenstate of the Schrödinger equation for the hydrogen atom is written as

$$
\psi_{n l m}(\boldsymbol{r}, t)=\chi_{n l}(r) Y_{l m}(\theta, \phi) e^{-i E_{n} t / \hbar}
$$

where

$$
\begin{aligned}
\chi_{n l}(r) & =-\left(\frac{2}{n^{2}}\right)\left[\frac{(n-l-1) !}{a_{0}^{3}[(n+l) !]^{3}}\right]^{1 / 2} \rho^{l} e^{-\rho / 2} L_{n+l}^{2 l+1}(\rho), \\
\rho & =\left(2 r / n a_{0}\right), \\
Y_{l m}(\theta, \phi) & =N_{l m} P_{l}^{|m|}(\cos \theta) e^{i m \phi},
\end{aligned}
$$

$L_{n+l}^{2 l+1}(\rho)$ are the associated Laguerre polynomials and $P_{l}^{|m|}(\cos \theta)$ are the associated Legendre functions 14]. Eq. A1 can then be written as

$$
\psi_{n l m}(\boldsymbol{r}, t)=N_{l m} \chi_{n l}(r) P_{l}^{|m|}(\cos \theta) e^{(i / \hbar)\left(\hbar m \phi-E_{n} t\right)},
$$

where $N_{l m}, \chi_{n, l}(r)$ and $P_{l}^{|m|}(\cos \theta)$ are real. Thus, the dynamics of the system in the Bohm description is provided by the quantum action

$$
S(r, \theta, \phi, t)=\hbar m \phi-E t
$$


The Eqs.(17) of motion are 20]

$$
\begin{aligned}
v_{r} & =\dot{r}=\frac{1}{\mu}\left(\frac{\partial S}{\partial r}\right)=0, \\
v_{\theta} & =r \dot{\theta}=\frac{1}{\mu r}\left(\frac{\partial S}{\partial \theta}\right)=0, \\
v_{\phi} & =\frac{1}{\mu r \sin \theta}\left(\frac{\partial S}{\partial \phi}\right)=r \sin (\theta) \dot{\phi} \\
& =\frac{m \hbar}{\mu r \sin \theta} .
\end{aligned}
$$

These can be integrated yielding

$$
r(t)=r_{0}, \quad \theta(t)=\theta_{0}
$$

and

$$
\phi(t)=\phi_{0}+\left(\frac{m \hbar t}{\mu r_{0}^{2} \sin ^{2} \theta_{0}}\right) .
$$

APPENDIX B: SECOND ORDER MOMENT IN THE STOCHASTIC QUANTUM MECHANICS

Let us here consider the calculation of the moments in SQM. According to the definition given in Eq.(33) for the state $|211\rangle$ (and for even $n$ ) one has

$$
\gamma_{\text {SQM }}^{(n)}=\left(\frac{e^{2} a_{0}^{2}}{64 \omega_{0}}\right) \int_{0}^{\infty} \omega^{n}\left(\frac{\omega_{0}}{\omega}\right)^{9 / 2} K_{1}\left(\sqrt{\frac{\omega_{0}}{\omega}}\right) d \omega .
$$

Using the change of variable $x=\sqrt{\omega_{0} / \omega}$, one finds

$$
\gamma_{\text {SQM }}^{(n)}=\left(\frac{e^{2} a_{0}^{2}}{32}\right) \omega_{0}^{n} \int_{0}^{\infty} x^{(6-2 n)} K_{1}(x) d x .
$$

This yields

$$
\begin{aligned}
\gamma_{\text {SQM }}^{(0)} & =12\left(e a_{0}\right)^{2} \\
\gamma_{\text {SQM }}^{(2)} & =\left(\frac{\left(e a_{0} \omega_{0}\right)^{2}}{16}\right)=\left(\frac{e^{4}}{16 \mu a_{0}}\right) .
\end{aligned}
$$

APPENDIX C: HYDROGEN ATOM INTERACTING WITH THE FIELD OF A PLANE ELECTROMAGNETIC WAVE

Let $H_{0}$ denote the Hydrogen atom hamiltonian and suppose to have an atom in one of his stationary eigen-states $(|N\rangle)$ at $t=t_{0}$

$$
\left|\Psi\left(t_{0}\right)\right\rangle=|N\rangle
$$


interacting with the electric field of a plane wave of frequency $\omega$ :

$$
\begin{aligned}
V_{i n t}(t) & =\boldsymbol{E}(t) \cdot \boldsymbol{d} \\
\boldsymbol{E}(t) & =E_{0} \boldsymbol{\epsilon}_{x} \cos (k z-\omega t)
\end{aligned}
$$

the elctric field component of the plane wave assuming that it is traveling in the $\mathrm{z}$ direction with momentum $k=\omega / c$ and $\boldsymbol{d}=e \boldsymbol{x}$ is the dipole operator.

The full hamiltonian is therefore:

$$
H=H_{0}+V_{\text {int }}(t)
$$

The quantity we would like to study is the total transition probability per unit time given that the system is initially in the state $|N\rangle$ at time $t=t_{0}$. Let us compute first the total transition probability $W_{N}^{\text {transition }}(t)$ at time $t$, given the initial condition in Eq. C1. At time $t$ the system will be in the state $|\Psi(t)\rangle$ obtained from the state $|N\rangle$ by application of the evolution operator (i.e. solving the Schrödinger equation for $\Psi$ ). Thus the probability $P_{N}(t)$ that at time $t$ the atom is still in the state $|N\rangle$ is given by:

$$
P_{N}(t)=|\langle N \mid \Psi(t)\rangle|^{2}
$$

Conservation of probabily requires that:

$$
P_{N}(t)+W_{N}^{(\text {transition })}(t)=1
$$

In this problem the interaction has an explicit time dependence and so it is useful to resort to the interaction representation:

$$
\begin{aligned}
|\Psi(t)\rangle & =U\left(t, t_{0}\right)\left|\Psi\left(t_{0}\right)\right\rangle \\
U\left(t, t_{0}\right) & =T e^{+\frac{i}{\hbar} \int_{t_{0}}^{t} d t^{\prime} V_{i n t}^{\prime}\left(t^{\prime}\right)} \\
V_{\text {int }}^{\prime}\left(t^{\prime}\right) & =e^{+\frac{i}{\hbar} H_{0} t^{\prime}} V_{\text {int }}\left(t^{\prime}\right) e^{-\frac{i}{\hbar} H_{0} t^{\prime}}
\end{aligned}
$$

where $\mathrm{T}$ stands for time ordering.

Thus the probability of being in the state $|N\rangle$ at time $\mathrm{t}$ is given by:

$$
P_{N}(t)=\left|\left\langle N\left|U\left(t, t_{0}\right)\right| N\right\rangle\right|^{2}
$$

In second order perturbation theory the amplitude of remaining in the state $|N\rangle$ at time $t$ is:

$$
\left\langle N\left|U\left(t, t_{0}\right)\right| N\right\rangle=1-\frac{i}{\hbar} \int_{t_{0}}^{t} d t^{\prime}\left\langle N\left|V_{i n t}^{\prime}\left(t^{\prime}\right)\right| N\right\rangle-\frac{1}{2 \hbar^{2}} \int_{t_{0}}^{t} \int_{t_{0}}^{t} d t^{\prime} d t^{\prime \prime}\left\langle N\left|T\left[V_{i n t}^{\prime}\left(t^{\prime}\right) V_{i n t}^{\prime \prime}\left(t^{\prime \prime}\right)\right]\right| N\right\rangle
$$


The dipole interaction we are considering does not contribute at first order since $\langle N|\boldsymbol{d}| N\rangle=0$ for any eigenstate of the hamiltonian $H_{0}$ (hydrogen atom). Thus:

$$
\begin{aligned}
\left\langle N\left|U\left(t, t_{0}\right)\right| N\right\rangle & =1-\Sigma_{N} \\
\Sigma_{N} & =\frac{1}{2 \hbar^{2}} \int_{t_{0}}^{t} \int_{t_{0}}^{t} d t^{\prime} d t^{\prime \prime}\left\langle N\left|T\left[V_{\text {int }}^{\prime}\left(t^{\prime}\right) V_{\text {int }}^{\prime \prime}\left(t^{\prime \prime}\right)\right]\right| N\right\rangle \\
P_{N}(t) & =1-2 \Re\left(\Sigma_{N}\right)
\end{aligned}
$$

Comparing this last Eq. with Eq. C5 one finds for the total transition probability $W_{N}^{\text {transition }}(t)=2 \Re\left(\Sigma_{N}\right)=2 \operatorname{Im}\left(i \Sigma_{N}\right)$ or:

$$
W_{N}^{\text {transition }}(t)=\operatorname{Im}\left\{+\frac{i}{\hbar^{2}} \int_{t_{0}}^{t} \int_{t_{0}}^{t} d t^{\prime} d t^{\prime \prime}\left\langle N\left|T\left[V_{\text {int }}^{\prime}\left(t^{\prime}\right) V_{i n t}^{\prime \prime}\left(t^{\prime \prime}\right)\right]\right| N\right\rangle\right\}
$$

Now let us insert the explicit form of the dipole interaction given in Eq. C2. The fact that the wave is assumed to be $x$-polarized selects the $x$ component of the dipole operator $\boldsymbol{\epsilon}_{x} \cdot \boldsymbol{d}=d_{x}=e x$ and:

$$
\begin{aligned}
V_{i n t}^{\prime}(t) & =e E_{0} x(t) \cos (k z-\omega t) \\
x(t) & =e^{+\frac{i}{\hbar} H_{0} t} x(0) e^{-\frac{i}{\hbar} H_{0} t}
\end{aligned}
$$

Inserting the above expression of $V_{\text {int }}(t)$ in Eq. C10 we also adopt the so called long wavelenght approximation which consists in neglecting the $z$ - dependence in the interaction. This is justified so long as $k a_{0} \ll 1$ being $a_{0}$ the Bohr radius. Thus one gets:

$$
W_{N}^{\text {transition }}(t)=\operatorname{Im}\left\{+\frac{i}{\hbar^{2}}\left(e E_{0}\right)^{2} \int_{t_{0}}^{t} \int_{t_{0}}^{t} d t^{\prime} d t^{\prime \prime}\left\langle N\left|T\left[x\left(t^{\prime}\right) x\left(t^{\prime \prime}\right)\right]\right| N\right\rangle \cos \left(\omega t^{\prime}\right) \cos \left(\omega t^{\prime \prime}\right)\right\}
$$

Then note that defining $\tau=t^{\prime}-t^{\prime \prime}$ and $t_{+}=t^{\prime}+t^{\prime \prime}$ one has:

$$
\begin{aligned}
\cos \left(\omega t^{\prime}\right) \cos \left(\omega t^{\prime \prime}\right) & =\frac{1}{2}\left[\cos (\omega \tau)+\cos \left(\omega t_{+}\right)\right] \\
\left\langle N\left|T\left[x\left(t^{\prime}\right) x\left(t^{\prime \prime}\right)\right]\right| N\right\rangle & =\left\langle N\left|T_{\tau}[x(\tau) x(0)]\right| N\right\rangle
\end{aligned}
$$

where $T_{\tau}$ stands for time ordering relative to the $\tau$ variable. Then define $t_{0}=-T / 2$ and $t=+T / 2$, change the integration variables according to $d t^{\prime} d t^{\prime \prime}=(1 / 2) d \tau d t_{+}$to obtain:

$$
W_{N}^{\text {transition }}(T)=\operatorname{Im}\left\{+\frac{i}{\hbar^{2}} \frac{\left(e E_{0}\right)^{2}}{4} \int_{-T}^{+T} \int_{-T}^{+T} d \tau d t_{+}\langle N|T[x(\tau) x(0)]| N\rangle\left[\cos (\omega \tau)+\cos \left(\omega t_{+}\right)\right]\right\}
$$

Now the time integration over the variable $t_{+}$is readily done:

$$
W_{N}^{\text {transition }}(T)=\operatorname{Im}\left\{+\frac{i}{\hbar^{2}} \frac{\left(e E_{0}\right)^{2}}{4} \int_{-T}^{+T} d \tau\left\langle N\left|T_{\tau}[x(\tau) x(0)]\right| N\right\rangle\left[2 T \cos (\omega \tau)+\frac{2}{\omega} \sin \left(\frac{\omega T}{2}\right)\right]\right\}
$$


And the total transition probability per unit time $w_{N}^{\text {transition }}$ (in the limit of infinite times) is extracted:

$$
w_{N}^{\text {transition }}=\lim _{T \rightarrow \infty} \frac{W_{N}^{\text {transition }}(T)}{T}=\operatorname{Im}\left\{+\frac{i}{\hbar^{2}} \frac{\left(e E_{0}\right)^{2}}{2} \int_{-\infty}^{+\infty} d \tau\left\langle N\left|T_{\tau}[x(\tau) x(0)]\right| N\right\rangle \cos (\omega \tau)\right\}
$$

Finally it is easily shown that:

$$
\left\langle N\left|T_{\tau}[x(-\tau) x(0)]\right| N\right\rangle=\left\langle N\left|T_{t} a u[x(\tau) x(0)]\right| N\right\rangle
$$

and hence:

$$
w_{N}^{\text {transition }}=\operatorname{Im}\left\{+\frac{i}{\hbar^{2}} \frac{\left(e E_{0}\right)^{2}}{2} \int_{-\infty}^{+\infty} d \tau e^{+i \omega \tau}\left\langle N\left|T_{\tau}[x(\tau) x(0)]\right| N\right\rangle\right\}
$$

Thus defining the time ordered "propagator" by:

$$
\alpha_{N}(\omega)=\frac{i}{\hbar} \int_{-\infty}^{+\infty} d \tau e^{+i \omega \tau}\left\langle N\left|T_{\tau}[x(\tau) x(0)]\right| N\right\rangle
$$

one writes the total transition probability per unit time as:

$$
w_{N}^{\text {transition }}=\frac{\left(e E_{0}\right)^{2}}{2 \hbar} \operatorname{Im}\left[\alpha_{N}(\omega)\right]
$$

This total transition probability when normalized to the flux of incident photons defines a total transition cross-section $\sigma_{\text {tot }}^{N}(\omega)$ (adsorption and possibly stimulated emission, if the state $|N\rangle$ is an excited state):

$$
\sigma_{\text {tot }}^{N}(\omega)=\frac{8 \pi \hbar \omega}{c E_{0}^{2}} w_{N}^{\text {transition }}
$$

or :

$$
\sigma_{\text {tot }}^{N}(\omega)=8 \pi \frac{\omega}{c} \frac{e^{2}}{2} \operatorname{Im}\left[\alpha_{N}(\omega)\right]
$$

On the other end we have shown, c.f. Eq. 23 that the imaginary part of $\alpha_{N}(\omega)$ is directly related to the spectral function (fourier transform) of the two-time correlation functions:

$$
\frac{\hbar}{2 \pi} \operatorname{Im}\left[\alpha_{N}(\omega)\right]=S_{N}(\omega)=\int_{-\infty}^{+\infty} d \tau e^{+i \omega \tau} \frac{1}{2}\langle N|[x(\tau) x(0)+x(0) x(\tau)]| N\rangle
$$

We therefore conclude:

$$
\sigma_{\text {tot }}^{N}(\omega)=8 \pi^{2} \alpha_{\mathrm{QED}}\left[\omega S_{N}(\omega)\right]
$$

$\alpha_{\mathrm{QED}}=e^{2} /(\hbar c) \approx 1 / 137$ being the fine structure constant.

[1] D. Bohm, Phys. Rev. 85, 166-179, (1952). 
[2] D. Bohm, Phys. Rev. 85, 180-93, (1952).

[3] D. Durr, S. Goldstein, and N. Zanghì. Journal of Stat. Phys. 116, (2004) 959-1055.

[4] K. Brendl, M. Daumer, D. Durr and N. Zanghì. Nuovo Cimento B 110, 737 (1995).

[5] D. Durr, S. Goldstein, and N. Zanghì. Journal of Stat. Phys. 67 (1992) 843-907.

[6] E. Nelson, Physical Review 150, 1079-1085, (1966).

[7] P. R. Holland, The Quantum Theory of Motion, Cambridge University Press,(1993).

[8] D. Hestenes, Am. J. Phys. 39 1028-1039 (1971). J. Math. Phys. 14 No.7 (1973), 893-905

[9] D. Hestenes, J. Math. Phys. 16, 556-572 (1975). D. Hestenes and R. Gurtler, J. Math. Phys. 16, 573-583 (1975).

[10] J. R. Fanchi "Parametrized Relativistic Quantum Theory” (Kluwer Dordrecht, 1993).

[11] J. R. Fanchi, Phys. Rev. A 34, 1677-1681 (1986). J.R. Fanchi, Found. Phys. 30, (2000), 1161-1189. J.R. Fanchi, Found. Phys. 31, (2001), 1267-1285.

[12] N. Redington, A. Widom and Y. N. Srivastava, Il Nuovo Cimento, 109B 1169-74 (1994).

[13] R. Balescu, Equilibrium and Non-equilibrium Statistical Mechanics. New York: Wiley \& Co., (1975).

[14] "Handbook of Mathematical Functions" Ed. M. Abramowitz and I. Stegun, Dover Publications, Inc. New York (ninth printing 1970).

[15] Levere Hostler, J. Math. Phys. 1 , 591 (1964).

[16] Robert R. Mapleton, J. Math. Phys., 2, 478, (1961).

[17] Koichi Mano, J. Math. Phys., 4, 522, (1963).

[18] I. S. Gradshteyn, I. M. Ryzhik, "Tables of Integrals, Series, and Products", Academic Press, New York, corrected and enlarged edition edited by Alan Jeffrey, (1980).

[19] M. J. W. Hall, J. Phys. A: Math. Gen. 37 (2004) 9549-9556.

[20] L. de Broglie, Nonlinear Wave Mechanics. Amsterdam: Elsevier., (1960). 